\title{
Subject Index Vol. 27, 1997
}

Academic outcome 63 Aicardi syndrome 100 Ambulation 40 Amniotic fluid 190 Anaplastic change 1 Angiogenesis 182 Apoptosis 246 Appendage 242 Astrocytoma 121 , 129 , 153 Autologous bone marrow rescue 230

Baclofen 40 Brachial plexus 19 Brain ischemia 182

- stem $12,92,153$

- neoplasm 12

- compression 223

tumor(s) 28,286

,- childhood 230

C57BL/6J mouse 176 Callosal agenesis 100 Calvarial vault remodeling 137 Catastrophic epilepsy 268 Cavernous angioma 92 Central nervous system 203 Cerebellar infarction 71 - mutism 28

Cerebellomedullary fissure 28 Cerebral edema 286

malformation 268

- palsy 40

revascularization 182 Cerebrospinal fluid 108

- flow dynamics 272 Cervicomedullary decompression 272

- junction 12

neoplasm 12 Chemotherapy 203 Chiari malformation 176

type I malformation 272

- II malformation 223 Chiasm 121

Child(ren) 71, 92, 105, 194 Choroid plexus cyst 100

- papilloma 100,108

Chronic subdural hematoma

292 CineMRI 149 Conus medullaris 257

Corpus callosotomy 260 Cosmetic outcomes 137 Cranial nerve rhizopathy 238 Craniosynostosis 78, 137

Dandy-Walker malformation

319 Delayed splotch mouse 176 Diencephalon 121, 129 Disconnection syndrome 260 Dura mater 182

Emergency 211 Encephalocele 214 Encephalopathy 230 Endoscopic shunt placement

319 Endoscopy 292 Enteric duplication 214 Ependymoma 84 Epicranial tumor 325 Epilepsy 260

- surgery 194,260,304,312 Excision 108

External carotid artery 182 Extremity 242

Filum terminale 257 Foramen caecum 246 Fourth ventricle 28, 176 Frontoethmoidal encephalocele

Ganglioglioma 203 Glioma(s) 1, 12,121 -, nasal 246 Gross total resection 34

Hemifacial spasm 238 Hemimegalencephaly 268 Hemispherectomy 268 Hemorrhage 92 Holocytochrome c-type synthetase 100 Hydrocephalus 63, 105, 149,

208,214 Hypervascular pediatric brain

tumors 296 Hypothalamus 121,

Ilizarov technique 78 Infant(s) 84,292

Interorbital hypertelorism 246 Intraventricular hemorrhage 63 Ion channels 286 ISG Viewing Wand 319

Lactate dehydrogenase 190

Magnetic resonance angiog-raphy 71,325

- - imaging 71,312

source imaging 304 Magnetoencephalography 304 Magnets 78

Meningitis 218 Meningoencephalocele 45 Microphthalmia, linear skin

defects syndrome 100 Microsurgery 19 Microvascular decompression

238 Molecular analysis, germ line

mutations 304 Myelomeningocele 190,223

Neoadjuvant chemotherapy

296 Nerve injury 19 Neural tube defect 176 Neurologic complications,

cancer 230 Neurotoxicity 230

Obstetrical palsy 19 Ochrobactrum anthropi 218 Optic chiasm 121,129 Organotypic culture 190 Osmolytes 286 Outlet 176

Pathogenesis 246 Pediatric brain tumors 1 
- intramedullary spinal cord

tumors 34

neurosurgery 108

Pediatrics 203

Pericardial tissue allografts 218 Personal computer 211 Pilocytic astrocytoma 1 Posterior fossa 71

- - shunt 319

Radiation therapy 203 Radical surgery 34 Refractory epilepsy 304 Reoperation 194

Sagittal synostosis 137 Shunt(s) 108,208

- failure 211 Side effect 45 Sinus pericranii 325 Software 211 Spinal cord 153

- lesions 34

- neoplasm 12

- dysraphism 214 Strip craniectomy 137 Stroke 71

Subdural grids and strips 312

Surgery 92

Surgical decompression 223

- resection 84

Survival 208

Tela choroidea 28

Teratoma, cervical 242

-, paraspinous 242

Tethered cord syndrome 257

Third ventriculocisternostomy, outcome 149

Three-dimensional reconstruction 312

Tonsillar coagulation 272

- herniation 272

Trapped fourth ventricle 319

Trauma 71

Traumatic brain injury 40

Tripterygium wilfordii 45

TSC1 gene 304

TSC2gene 304

uberous sclerosis complex 304

Tumor 153

Vascular malformation 105 Ventriculoperitoneal shunt,

outcome 63 Ventriculoscope 149 Vermis 28

Vertebral artery dissection 71 Volume regulation 286

\section{KARGEfc}

Fax+ 416130612 34 E-Mail karger@karger.ch www.karger.com
(C) 1998 S. KargerAG, Basel 